\title{
Hierarchical spatial reasoning theory and GIS technology applied to the automated delineation of administrative boundaries
}

\author{
Serryn Eagleson ${ }^{\mathrm{a}}$, Francisco Escobar ${ }^{\mathrm{b}}$, Ian Williamson ${ }^{\mathrm{a}, *}$ \\ ${ }^{a}$ Department of Geomatics, The University of Melbourne, Melbourne, Victoria 3010 Australia \\ ${ }^{\mathrm{b}}$ Department of Geospatial Science, RMIT University, GPO Box 2476V, Melbourne, \\ Victoria 3001 Australia
}

Accepted 8 October 2001

\begin{abstract}
Throughout history, humankind has segmented and delineated the geographic environment in various ways to support administrative, political and economic activities. To date, the majority of spatial boundaries have been constructed in an uncoordinated manner with individual organisations generating individual boundaries to meet their own specific needs. As a result of this lack of coordination, there is a fragmentation of information over a series of boundary units, which not only limits the potential uses for data collected, but also the scope of analysis possible between boundary layers. The proposed solution outlined in this research involves the reorganisation of the spatial environment based on Hierarchical Spatial Reasoning (HSR) and the application of a GIS-based algorithm for the automated delineation of boundaries. By using this approach, it is expected that administrative boundaries can be formed through the aggregation of smaller units. This proposed system is focussed towards facilitating rapid and efficient cross analysis of data sets. (C) 2002 Elsevier Science Ltd. All rights reserved.
\end{abstract}

Keywords: Geographical information system; Spatial data infrastructure; Administrative boundaries; Hierarchical spatial reasoning (HSR)

\section{Introduction}

The ability to efficiently exchange and integrate data is crucial to the successful future of the geospatial sector. One of the major problems limiting the integration,

* Corresponding author. Tel.: +61-3-8344-4431; fax: +61-3-9347-4128.

E-mail address: ianpw@unimelb.edu.au (I. Williamson). 
comparison and transfer of data is the arrangement of administrative boundaries. In the majority of cases these administrative boundaries have been created by individual agencies to meet their specific needs, usually with very little inter-agency coordination. Due to this lack of co-ordination, current technologies for analysing geospatial information, such as GIS, cannot provide accurate results. With GIS currently being utilised across many fields including health, planning and marketing, the inherent problems with data analysis are becoming increasingly apparent.

One of the many roles of a Spatial Data Infrastructure (SDI) is to facilitate the integration of spatial data. It is well recognised that within a SDI a number of administrative boundary systems exist. The focus of this research is to test the possibility of integrating the needs of at least two agencies into one common spatial hierarchy. For this purpose the boundary systems focussed on are the Australian Bureau of Statistics (ABS), Census Collection Districts (CCDs) and the Australia Post postal areas. As a result of the cooperation and support for the research received from both the ABS and Australia Post. The research is also being supported by two Federal Government grants.

The methodology adopted in this research utilises the principles of Hierarchical Spatial Reasoning (HSR) theory and incorporates them into the automated design of administrative boundaries. HSR is defined by Car (1997) as part of the spatial information theory that utilises the hierarchical structuring of space and reasoning. People often break down problems into smaller problems to reduce their complexity. However, only a relatively small number of HSR models have been implemented in the GIS environment (Car, 1997; Frank \& Timpf, 1994; Glasgow, 1995). The benefits of furthering HSR theory to the organisation of administrative polygon layers are vested in the properties inherent within HSR described later in the paper.

This research is described in the following five sections. The first section outlines the significance of administrative boundaries and the major problems presented by the non-coterminous alignment of boundaries. The second section briefly outlines the theoretical framework to be used within the project for the automated delineation of administrative boundaries. The third section details the formalisation of the framework in the form of a prototype, whilst the fourth and fifth sections comment on the future developments and the conclusions of the research, respectively.

\subsection{Administrative boundaries within the SDI}

The SDI is an initiative intended to create an environment which enables a wide variety of users access to complete and consistent data sets in an easy and secure way. An SDI comprises policy, fundamental datasets, technical standards, accesses networks and people. The nature of the SDI is both dynamic and complex (Rajabifard, Escobar, \& Williamson, 2000). Increasingly, to support SDI policy, GIS technology is utilised for the storage, display and analysis of geospatial information. This trend has been widely supported by users of spatial information, as GIS provides an array of analytical tools to facilitate the decision making process. In many instances, the data used in analysis is attached to polygons defined by administrative boundaries. 


\subsection{The problem}

Increasingly, data referenced to administrative polygons, such as postal areas, CCDs, health districts and police districts, are required in a diverse range of applications. However, due to the uncoordinated delineation of these boundaries, accurate cross analysis between them is restricted. Fig. 1 illustrates the current situation in an Australian state where each agency establishes a different size and shape of spatial unit, based on their individual requirements. In turn, each organisation aggregates these boundaries in a hierarchical fashion to cover their area of interest. Data aggregation is possible for each organisation; however, under the current system, additional methods must be employed to facilitate cross analysis between organisations.

Health service planning in Victoria, Australia provides a classic example of the restricting nature of non-coterminous boundaries within the GIS environment. Medical institutions often attach data to postal areas, while demographic data is attached to CCDs. As a result, accurate cross analysis between demographic and health statistics is virtually impossible. The analysis of child immunisation statistics demonstrates this difficulty. The records detailing the number of children immunised are attached to postal areas, to calculate if this number is below or above the average population data is required. Thus, without additional information it is impossible to establish if child immunisation rates, within a particular postal area, are below or above an acceptable limit, restricting the accurate planning of health services.

Many authors have highlighted the relevance of investigating the problem of data integration between incompatible boundary systems. These authors include:

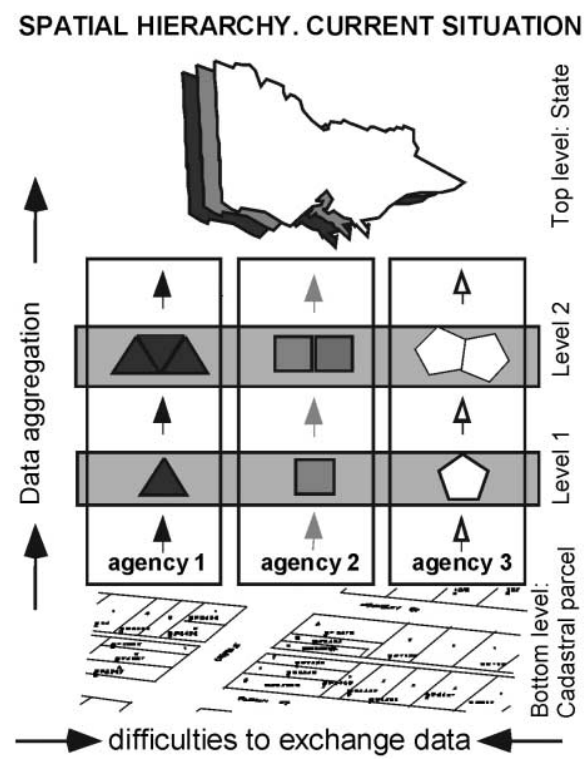

Fig. 1. Current hierarchical spatial structures: the problem. 
Bracken and Martin (1989), Eagleson, Escobar, and Williamson (2000), Fischer and Nijkamp (1993), Huxhold (1991), Openshaw, Alvandies, and Whalley (1998). The significance of investigating this problem has been further emphasised in various forums in Australia, particularly the Victorian Geospatial Information Reference Group (GIRG) 1998 (http://www.giconnections.vic.gov.au/content/groups/girg/ archive.htm) and the First Symposium of GIS in Health, Melbourne (Escobar, Williamson, Waters, Green, \& Rudd, 1997). These authors and forums suggest if spatial analysis techniques are to reach their full potential the data framework within SDIs should incorporate the development of compatible spatial units (Eagleson et al., 2000).

Due to the restricting nature of these administrative boundaries a theoretical framework for the coordinated design of administrative boundaries within the SDI is described in the following section.

\section{Prototype development}

\subsection{Administrative boundary design}

In the past, administrative boundaries have been designed with little thought to their integration capabilities. Instead, individual agencies have constructed individual boundaries in isolation without a theoretical framework. One of the contributing factors to this problem is the fact that boundary systems evolved before GIS appeared. Therefore, many boundary systems have been drafted on hard copy maps and digitised for incorporation into GIS. Prime examples of this evolution of boundaries from analogue to digital format are the postal areas and CCDs of Australia. However, with advances in technology, the availability of digital data and growing awareness of problems associated with data aggregation, new structured mechanisms for boundary delineation are required.

\subsection{Automated boundary design}

Previous research into the automated design of administrative boundary systems can be subdivided in two groups: those operating by redistricting existing areas and those based on the aggregation of existing smaller administrative units. In redistricting the majority of research has taken place in the United States and involves the reallocation of electoral district boundaries. The most basic redistricting methods are interactive. The user selects a geographic unit on the screen and then issues a command to assign it to a district or transfer it from one district to another. The system offers immediate feedback on the political and demographic consequences of each move. Automated algorithms for redistricting have been developed using techniques such as simulated annealing (Browdy, 1990; Macmillan \& Pierce, 1994). The theory behind simulated annealing is to prevent the system stopping at premature local optima, increasing the chance that it will eventually find the global optimum. 
In contrast to redistricting methods, current boundary aggregation techniques are based on the reaggregation of existing units into new boundaries more suitable for specific analysis techniques. For example, Openshaw (1977) devised the automated zone design program (AZP) for investigating the Modifiable Area Unit Problem (MAUP). During the mid 1990s using new technology, digital data and improved algorithms, AZP was further refined and extended forming the Zone DEsign System (ZDES; Openshaw \& Alvandies, 1999; Openshaw \& Rao, 1995). These zone design systems have been designed to allow the data analyst the freedom to start with data at one scale and then reaggreagate it to create a new set of regions designed to be suitable for a specific purpose independent of the collection boundaries used (Openshaw \& Rao, 1995).

Both the redistricting and aggregation approaches to boundary design utlise existing boundary units for the creation of new boundary units. However, if these initial boundaries are not designed as layers within a hierarchy, the problem of data integration between overlapping polygons remains. The prototype designed in this research aims to provide a systematic and rigorous method for the design of new administrative boundaries. Once established, the spatial hierarchy is designed to incorporate data stored at the lowest level of the hierarchy such as address points through to state and national administrative boundaries. As a result this approach is expected to facilitate the coordinated collection and dissemination of data both vertically up and horizontally across all levels of the spatial hierarchy. It is an approach which has particular potential in jurisdictions or countries, which have well-developed cadastral systems, with the associated digital maps of parcel boundaries. Such jurisdictions include western Europe, Canada, Australia and New Zealand.

\subsection{Hierarchical spatial reasoning}

Conceptually a spatial hierarchy consists of many levels with the higher levels in the hierarchy being aggregations of smaller units. This hierarchical concept is demonstrated by Coffey (1981), who utilises the set of triangles as shown in Fig. 2 to illustrate the nature of hierarchy in terms of space. As illustrated below, triangle $\mathrm{ABC}$ consists of four sub triangles, one of which is ADF, which in turn consists of four smaller triangles. This pattern of subdividing space into smaller units is repeated continuously down to the smallest spatial unit. This repetitive breakdown is more formally referred to as a spatial hierarchy (Coffey, 1981). Although spatial hierarchies are designed using principles - to break complex tasks into sub tasks or areas - relationships between levels within the hierarchies are complex.

In the past much research has focussed on the properties of two-dimensional hierarchical structures to model networks, such as road and drainage systems. This research, however, aims to utilise the three properties (Whole-Part, Janus Effect and Near Decomposability) inherent to hierarchies, and adopt them in boundary design. These properties provide an insight into the way that each element within a hierarchy interacts with each other and also with the whole system (Car, 1997). 


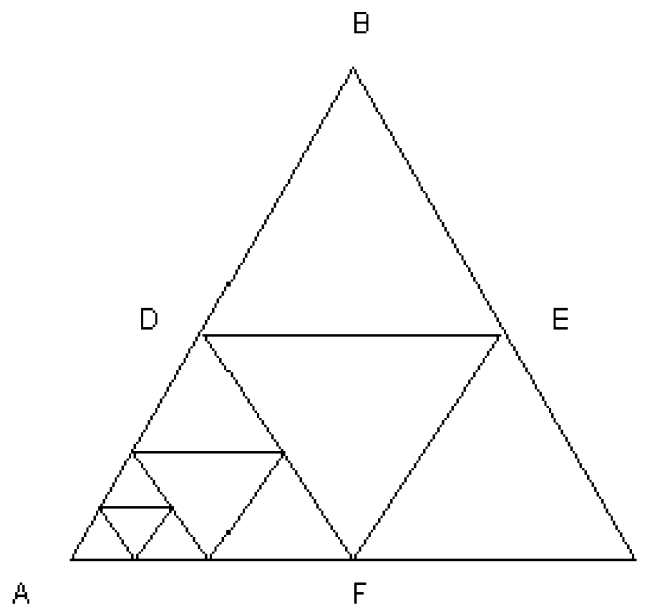

Fig. 2. Illustrates the concept of a spatial hierarchy through the use of triangles as a key spatial unit (source: Coffey, 1981, adapted).

\subsection{Proposed solution - HSR theory applied to administrative polygons}

Once the individual agencies' business rules are defined, a model can be established to hierarchically organise spatial units. It is anticipated that these units will meet requirements of GIS users whilst remaining effective as administration boundaries to each of the agencies involved. Fig. 3 adopts an abstract view where three agencies with different spatial needs have aggregated spatial units in a hierarchy. Each layer of the hierarchy is established automatically and in compliance with the geospatial requirements of the agency. The proposed solution is based on the aggregation of units in accordance with HSR theory. To be consistent with HSR theory and the geospatial requirements of administrative agencies the algorithm devised must have the ability to:

1. automatically subdivide the territory in compliance with the geospatial requirements stipulated by the relevant agency; and

2. to be recursive and reapplied to the outputs in order to produce new levels of the hierarchy, this characteristic will guarantee compliance with HSR theory.

In the past, research has been conducted into maximising the efficiency of computational processes by using hierarchies to break complex tasks into smaller, less complex, tasks. Due to the complexities of spatial entities there are often many alternatives to the simplest problems. Therefore the objective of HSR theory is not to obtain an 'optimum' result but one which meets user specifications at each level requiring the least effort (Car, 1997; Timpf \& Frank, 1997). Through the application of HSR theory within this research, spatial boundaries of different agencies are aggregated from a common base layer into a coordinated hierarchical system of functional administrative boundaries. Additionally it is possible to exchange data between the agencies at each stage of the hierarchy. 


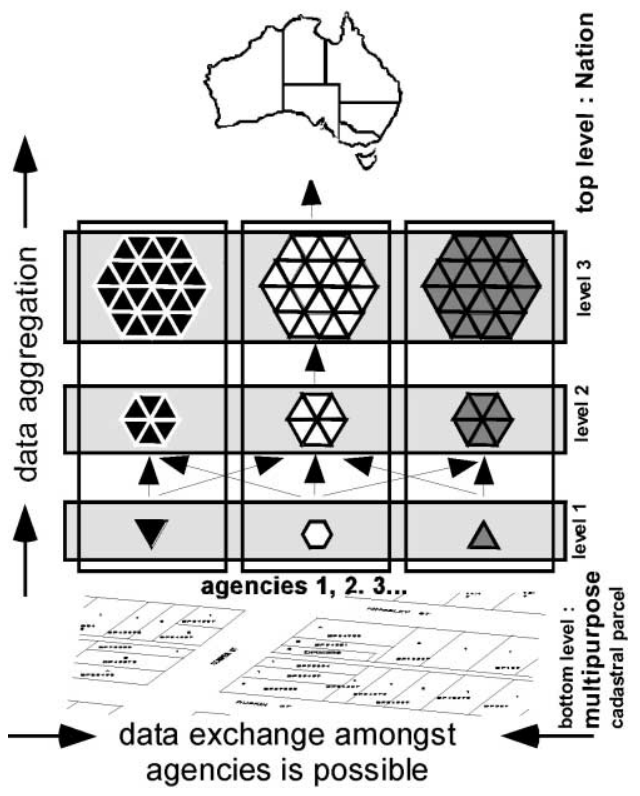

Fig. 3. Future hierarchical spatial structures; the solution.

\subsection{Strengths of the proposed system}

The importance of a strong theoretical framework for the development of a spatial hierarchy cannot be over emphasised. This theoretical solution outlined earlier provides a systematic, recursive and rigorous method to the task of boundary delineation. In the past, postal area and CCD boundaries of Australia have been constructed in an uncoordinated manner. Using the method proposed in this research, a flat non-hierarchical system can be transformed using business rules or constraints in the development of new administration boundaries. Applied by various organisations, this method can be used to delineate boundaries in an environment where each of them is able to establish their own business rules and apply them in a coordinated and consistent manner.

In automating this approach for administrative boundary allocation, there is an added advantage of being fast, repeatable and flexible. The flexibility of the system enables additional parameters such as size, density of households, centres of community interest and shape to be incorporated into the boundary design process. The ability of the system to incorporate additional parameters will enable it to meet the requirements of users in different agencies and/or different regions with different needs. Being repeatable means that agencies will be able to adopt similar methods for the design of administrative boundaries, thus limiting subjectivity. Additionally, this method will aid in the comparison of datasets over time as each set can be broken down to the base layer. 


\subsection{Modifiable Area Unit Problem (MAUP)}

A classic problem associated with the design and display of boundaries is the MAUP. The MAUP is "a form of ecological fallacy associated with the aggregation of data into areal units for geographical analysis. This aggregated data is then treated as individuals in analysis," (Openshaw \& Taylor, 1981). The MAUP can be divided into two parts, level of aggregation, and zoning configuration (Fotheringham \& Wong, 1991). This problem is fundamental in the display of demographic data as the information people perceive can be altered by the size, shape, and scale that is used for display (Fotheringham \& Wong, 1991). In the past, because boundaries were assumed fixed, researchers had to use whatever boundaries were available (Openshaw et al., 1998). Consequently the user has little, if any, control over the MAUP.

However, as Openshaw and Taylor (1979) explain, it is now possible for data users to exert some influence over or minimise the impact of the MAUP. To achieve this, it is recommended that analysts:

1. start from the smallest divisions available, or the smallest they can process;

2. aggregate these in a fashion relevant to their investigation; and

3 . assess the repeatability of their results for several aggregations.

It is expected that the proposed hierarchical reorganisation model will allow agencies and GIS analysts to have greater flexibility over the level of aggregation and zoning configuration of spatial units used in analysis. Although this does not completely solve the MAUP it does allow analysts to exert corrective influence over the problem rather than ignoring it as in the past.

The implementation of a spatial hierarchy requires a technical solution. The following section outlines the study region, agencies and constraints utilised within the prototype development phase.

\subsection{Prototype development region}

The State of Victoria, Australia, has been identified as a suitable test base for the development of this research. The following points outline the specific benefits associated with using Victoria, Australia, for the application of new boundary delineation methods.

1. CCD and postal area boundaries are readily available, in digital form, to organisations for data collection and analysis within Australia.

2. Australia is in the process of establishing a complete Australian SDI (ASDI) policy. To facilitate the objectives of the ASDI projects such as the AUSLIG partnership programme (http://www.auslig.gov.au/asdi/grants.htm) have acknowledged that a well-structured spatial hierarchy is required.

3. Australia is undergoing continuous change with rapid expansion causing boundaries to be reassessed at regular intervals. Therefore clearly defined methods must be in place to ensure boundaries are delineated according to established criteria. 
4. Australia has vastly different regions from the densely populated to vast expanses of low population. As a result, testing models on these different regions indicates the research will be adaptable to other countries that are in the process of developing or reengineering administrative boundaries.

5. Recognising the importance of administrative boundary integration. Land Victoria, the State government agency responsible for core state data sets, has supplied cadastral and topographic digital data sets for prototype development and testing.

6. Two key agencies, which are responsible for establishing and maintaining administrative boundaries, ABS and Australia Post, are supportive and are cooperating with the research.

\subsection{Administrative boundary constraints}

Although it is well recognised that a number of boundary systems exist within Victoria, the focus of this research is to test the concept of integrating the needs of two selected agencies into one common spatial hierarchy. Therefore for this purpose the boundary systems selected for investigation are the CCD and postal areas. These boundaries have been selected due to their national coverage and acceptance in both the geospatial and public sectors within Australia.

To gain an insight into the role of boundaries within each agency, interviews were conducted with key personnel in Australia Post and the ABS. In this instance, the stated purpose of the boundaries is to support the administrative functions of two agencies. In particular, the focus of the agencies is on efficient delivery and collection mechanisms as well as the display of census related data. To be successful at achieving the objectives of the administrative boundaries, there are a number of constraints (otherwise known as 'business rules') that must be met. These constraints were based on the requirements of Australia Post and the ABS and include:

1. The preservation of topographic barriers. Examples of barriers include large rivers and roads that may obstruct delivery mechanisms. Additionally, these boundaries often divide different community groups and these differences are imperative to many planning activities and should where possible be preserved.

2. In order to preserve confidentially, the ABS state that each CCD unit must contain a range of between 150 and 200 households (ABS, 1996).

3. To facilitate delivery mechanisms it is important that the boundaries are in alignment with the road network and are identifiable on the ground. Using the meshblock for the aggregation assures this requirement is met.

4. To ensure uniformity across the area it is important that the boundaries are contiguous and provide complete coverage across the area without gaps or overlaps.

5. Although there is no formal definition of boundary shape it was decided that the boundaries should be constructed in a manner that enabled them to be compact. To ensure the boundaries established are compact the model tests 
each boundary based on the circularity index devised by Tomlin (1992). (Eagleson et al., 2000).

\section{Technical formalisation and implementation}

This section details the development of the prototype. Initially detailed is the data compilation followed by an outline of the algorithm developed within the research.

The development of input data is relatively straightforward. For the algorithm to work, each polygon used on the input layer must contain three key attributes. The first is the individual polygon identification (ID) number, the second is the number of address points/households and the third is the topographic region ID (illustrated in Fig. 4). Preparing the data layer involves the integration of each the address, road centreline and topographic data sets according to the following sequence of tasks:

1. build polygon topology of the road network forming meshblocks;

2. build polygon topology of the major infrastructure and major topographic features - this layer will act as a constraint in the program development;

3. union address data with the meshblock layer - the resulting meshblock layer contains the number of households per meshblock; and

4. union of the three coverages above.

Finally, the input dataset is a meshblock layer with each block attributed with the three fundamental attributes (ID, number of address points and a unique topographic region ID).

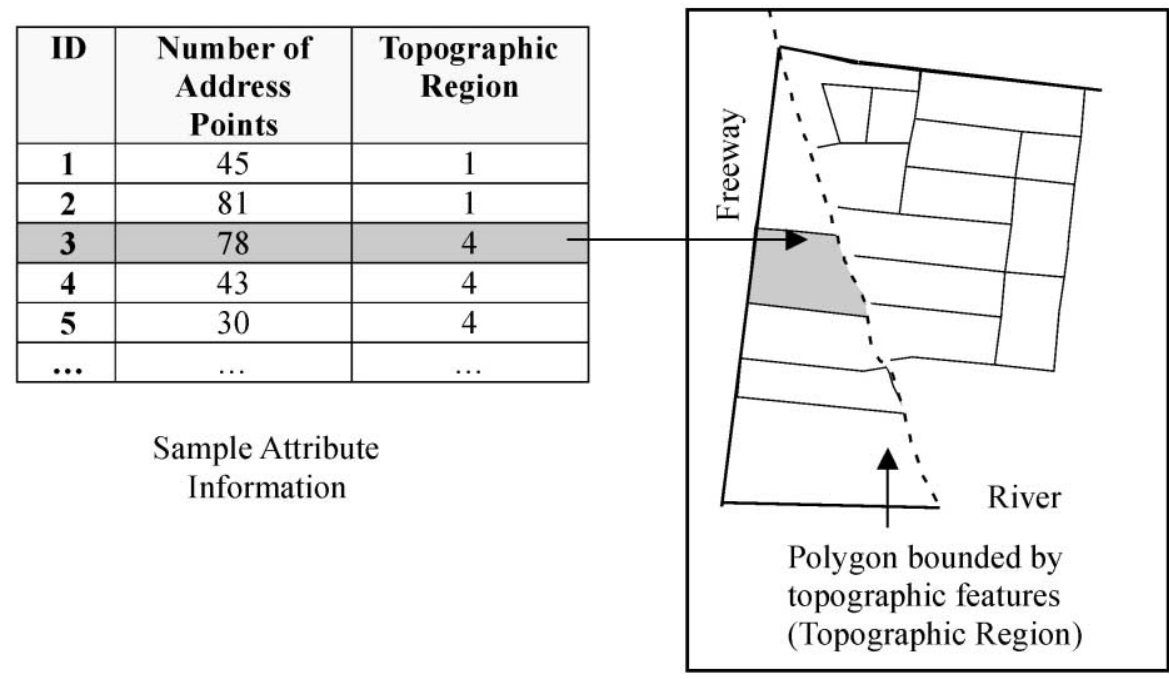

Graphic Representation

Fig. 4. Illustration of input data. 


\subsection{The algorithm}

\subsubsection{User perspective}

After entering the GIS and displaying the input data to be used, the user then calls the algorithm, either through an extension or by loading scripts into the view. Any relevant input such as the confidentiality range or degree of circularity required can be input manually. The algorithm runs and when finished displays the new administrative boundary layer.

\subsubsection{Algorithm mechanics}

The mechanics of the algorithm are hidden from the user. Behind the screen the algorithm runs through a series of routines ensuring the requirements of agencies are met. The following seven steps outline each of the decisions and processes utilised within the algorithm.

Step 1: Select a topographic region to be segmented (this polygon will be known as region $x$ ).

Step 2: Based on minimum centroid coordinates select a seed meshblock, within region $x$, and initialise a confidentiality counter to the number of addresses contained within the meshblock.

Step 3: Within region $x$ select all polygons contiguous with the seed selected in step 2.

The algorithm then assesses which of the adjacent polygons (within region $x$ ), when joined with the initial seed polygon, will yield the most compact shape. The method used for assessing shape is based on the reason of circularity index. This index has been adapted from Tomlin (1992), and is broken into the following components detailed in Eq. (1).

$$
\mathrm{RC}=\sqrt{\frac{\mathrm{SZ}}{\mathrm{SC}}}
$$

where: $\mathrm{RC}=$ reason of circularity; $\mathrm{SZ}=$ surface of the current zone; $\mathrm{SC}=$ surface of circle having the same perimeter (source: Tomlin, 1992).

As the reason of circularity approaches one, the polygon in question approaches a circular shape. As the reason of circularity approaches zero the polygon in question approaches a linear shape. Therefore, once each adjacent polygon is tested along with the initial polygon against the reason of circularity. The polygon with the highest reason of circularity is selected.

Step 4: Dissolve the boundary between the seed meshblock and the meshblock selected in Step 3. Update the value of the confidentiality counter (number of combined address points). This new unit becomes the seed.

Step 5: Repeat Steps 3 and 4 until the frequency required for confidentiality is reached. The overall circularity of the resulting boundary is then calculated. If the overall circularity of the new administrative unit falls below the reason of circularity threshold specified by the program operator a warning message is assigned to the polygon and stored in the attribute table. 
Step 6: Repeat Steps 2-5 until all the meshblocks within region $x$ have been aggregated.

Step 7: Repeat the process from Step 1 until all regions are processed.

This process is illustrated graphically in Fig. 5.

Fig. 6 illustrates the progression of aggregation from mesh block units through to administration units created automatically using the prototype developed in the research.

In summary, the algorithm takes an initial seed meshblock, and then assesses which of the contiguous polygons are the most suitable for aggregation. The chosen polygon is aggregated with the seed. This process is repeated until the confidentiality range input by the user is reached. The algorithm has been formalised using Avenue, an object-oriented programming language that operates under ArcView, the desktop GIS software developed and distributed by ESRI (Eagleson et al., 2000). For further information about the algorithm and to download sample scripts and data used

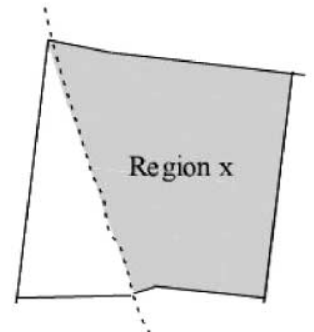

Step 1 - Region $\mathrm{x}$ selected

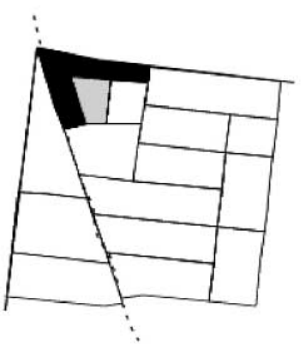

Step $3 b-$ Most compact contiguous polygon selected

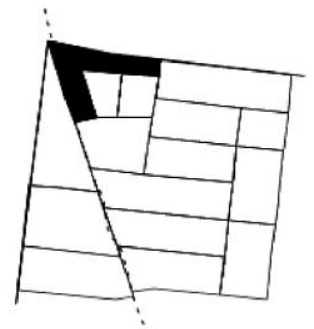

Step 2 - Seed polygon selected

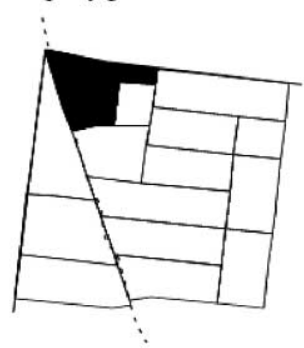

Step 4 - Dissolve the common boundary, forming the new seed.

Step 5-Repeat Step 2 and 3 using the new seed

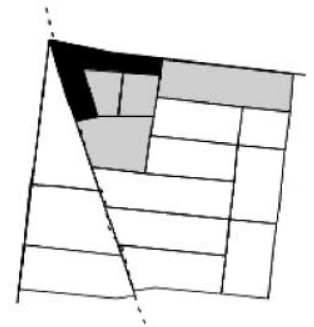

Step $3 \mathrm{a}-$ Contiguous polygons within Region $\mathrm{x}$ selected

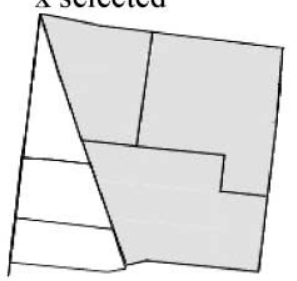

Step 6 - Repeat steps $2,3,4$ and 5 until all the meshblocks within region $\mathrm{x}$ have been aggregated.

Step 7 - Repeat the process from Step 1 until all the regions processed

Fig. 5. Graphical illustration of Steps 1-7 undertaken by the algorithm. 


\section{Data Input}

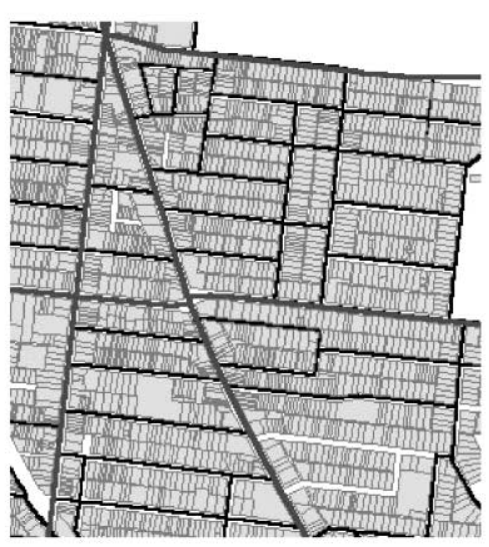

Topographic Barriers
Cadastre
$\square$ Meshblock

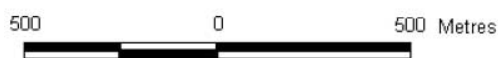

Data Output

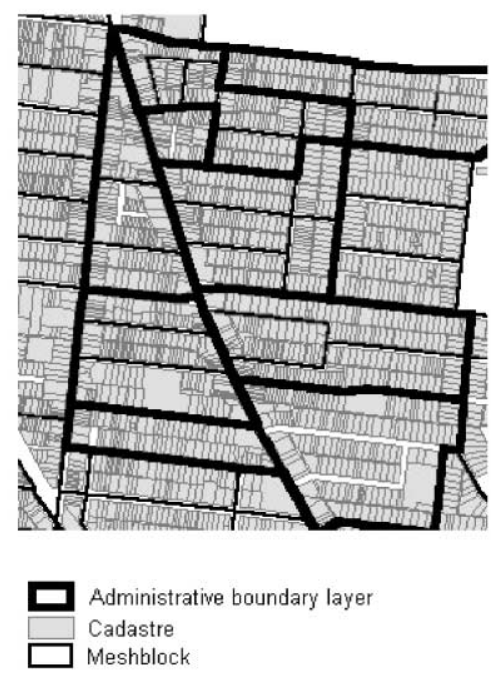

Fig. 6. Sample results.

within the project please refer to the project website (http://www.sli.unimelb.edu.au/ AUSLIG/).

\subsection{Limitations}

The aim of this research project is to develop new methods through which space can be divided into administrative boundaries in a structured manner. In undertaking this research it has become clear that it is possible to align administrative boundaries based on HSR theory. This was further supported by the development of a prototype for the automated delineation of administration boundaries using GIS. Limitations and areas for further improvement do, however, exist within each of the input data and algorithm development phases.

Throughout this project, it has been assumed that the input data is always available, complete and without error. However in many regions there are problems inherent within the digital data. For instance, many creeks and rivers are not continuous and as a result when the data set is cleaned the creeks appear as meshblocks. Additionally, the meshblock may not always be the optimum base unit and in some instances where strata titles exist in urban regions, there may be a requirement to split the meshblocks into smaller units. Therefore an additional test program may be required for testing instances where it is practical to split the meshblocks. In some instances the meshblock is dynamic and if altered may result in unstable boundaries. 
Due to the undefined requirements for the final administrative boundary shape the index of circularity has been introduced to simply guide the overall shape and warn users if the boundary falls below a predetermined threshold. However, as shape is important, it needs to be clearly defined by the agencies and methods established that correct boundaries which do not conform to the shape requirements.

\section{Future developments}

Future research lies in the realm of program testing and implementing the GIS prototype for automatic boundary allocation, based on the theory of HSR. In particular, issues need to be overcome in detailing criteria for rural boundaries where the methods for establishing administrative boundaries is often vastly different from metropolitan areas. For example in rural regions, roads often unite rather than segment communities as assumed in metropolitan areas. Also, adaptations to the model are required to suit social issues in boundary delineation such as the identity of place and spatial cognition.

With the increased demand for geospatial information, it is proposed that the realignment of administration boundaries, based on HSR, will overcome many of the present data fragmentation issues. However, to achieve this hierarchy of administration boundaries the theory of HSR requires further development to incorporate the complexities of polygon structures.

It may also be possible for the application of the prototype to be expanded to a wide array of commercial applications. For example, it is recommended that businesses requiring boundaries employ techniques such as the one outlined in this paper to become part of the spatial hierarchy. This would facilitate businesses, requiring boundaries for the analysis of market trends and the functional product distribution, to set the criteria for their boundaries inline with agency boundaries efficiently. As part of the spatial hierarchy this would allow businesses to cross analyse data with other agencies, such as the ABS, further enhancing their marketing and distribution techniques.

\section{Conclusion}

Current problems associated with non-coterminous boundary systems are identified in the paper. In response to these problems the primary objective of this research was to develop a theoretical framework for the effective future design of administrative boundaries. To achieve this objective a number of research phases have taken place. These include:

1. The development of a theoretical framework. This framework builds upon the properties of HSR theory, administrative agency constraints, whilst considering the planning in the modern city, and providing a partial resolution to the MAUP. 
2. Establishing the requirements and use of spatial boundaries by relevant agencies. In particular, focussing on boundaries assigned by Australia Post and the ABS.

3. The construction of a GIS prototype for the automatic allocation of administration boundaries. The implemented prototype outlined offers a solution to the problem of boundary delineation and provides the means for accurate data exchange between agencies. It facilitates a quick, objective and improved method to administrative boundary subdivision.

In conclusion, this research aims to complement effective data management strategies so that the full potential of spatial data can be realised.

\section{Acknowledgements}

The authors wish to gratefully acknowledge the support of Land Victoria (LV), of the Victorian Government, Land and Property Information (LPI) of the New South Wales Government, Australian Research Council (ARC) (Grant No. S499714), Australian Surveying and Land Information Group (AUSLIG), and the members of the Spatial Data Infrastructure and Land Administration Research Group at the Department of Geomatics, The University of Melbourne, in the preparation of this paper and the associated research. However, the views expressed in the paper are those of the authors and do not necessarily reflect the views of LV, LPI, ARC, AUSLIG and the Department of Geomatics.

To a large degree the success of this project rests with the agencies involved with the design of CCD and postal area boundaries. Particular mention should be made of Chris Reynolds, Manager Address Management Centre of Australia Post and Frank Blanchfield, Director Geography Section of the Australian Bureau of Statistics (ABS). Their continued support for the project is appreciated.

The authors also wish to acknowledge the encouragement and support of Professor Don Grant (former Surveyor General of New South Wales, Australia, and Chairman, Public Sector Mapping Agencies of Australia) in the investigations into spatial hierarchy problems.

\section{References}

Australian Bureau of Statistics (ABS). (1996). Statistical geography (Vol. 2: census geographic areas Australia). Canberra: Commonwealth of Australia.

Bracken, I., \& Martin, D. (1989). The generation of spatial population distributions from census centroid data. Environment and Planning A, 21, 537-544.

Browdy, M. (1989). Simulated annealing - an improved computer model for political redistricting. Yale Law and Policy Review, 8, 163-179.

Car, A. (1997). Hierarchical spatial reasoning: theoretical consideration and its application to modeling wayfinding. PhD thesis, Department of Geoinformation, Technical University, Vienna. 
Coffey, W. J. (1981). Geography: towards a general spatial systems approach. New York: Methuen.

Eagleson, S., Escobar, F., \& Williamson, I. P. (2000). Hierarchical spatial reasoning applied to the automated design of administrative boundaries using GIS. In URISA. Florida, Orlando: 18-23 August (CD-ROM).

Escobar, F., Williamson, I. P., Waters, J., Green, G. H., \& Rudd, C., (1997). The role of GIS in the management of primary health care services. AURISA 97-the 25th annual conference of the Australasian urban and regional information systems association (pp. 19-21). Christchurch, New Zealand.

Fischer, M., \& Nijkamp, P. (1993). Geographic information systems, spatial modeling and policy evaluation. Berlin: Springer-Verlag.

Fotheringham, A., \& Wong, D. (1991). The modifiable areal unit problem in multivariate statisical analysis. Environment and Planning A, 23, 1025-1044.

Frank, A. U., \& Timpf, S. (1994). Multiple representations for cartographic objects in a multi-scale treean intelligent graphical zoom. Computers and Graphics; Special Issue on Modelling and Visualization of Spatial Data in GIS, 18(6), 823-829.

Glasgow, J. (1995). A formalism for model-based spatial planning. In A. Frank, \& W. Kuhn (Eds.), Spatial information theory - a theoretical basis for GIS (pp. 501-518). Berlin: Springer.

Huxhold, W. (1991). An introduction to urban geographic information systems. New York: Oxford University Press.

MacMillan, W., \& Pierce, T. (1991). Optimizarion modelling in a GIS framework: the problem of political redistricting. In S. Fotheringham, \& P. Rogerson (Eds.), Spatial analysis and GIS. London: Taylor \& Francis.

Openshaw, S. (1977). A geographical solution to scale and aggregation problems in region-building, partitioning, and spatial modelling. Trans. Inst of British Geographers New Series, 2, 459-472.

Openshaw, S., \& Alvandies, S. (1999). Applying geocomputation to the analysis of spatial distributions. In P. A. Longley, M. F. Goodchild, D. J. Maguire, \& D. W. Rhind (Eds.), Geographic information systems: principles and technical issues 2nd ed. New York: John Wiley and Sons Inc. 267-282.

Openshaw, S., Alvandies, S., \& Whalley, S. (1998). Some further experiments with designing output areas for the 2001 UK census School of Geography, University of Leeds. Available: http://www.geog.soton.ac.uk/research/oa2001/resources.htm (access date: 12 November 2000).

Openshaw, S., \& Rao, L. (1988). Algorithms for reengineering 1991 census geography. Environment and Planning A, 27, 425-446.

Openshaw, S., \& Taylor, P. J. (1979). A million or so correlation coefficients: three experiments on the modifiable areal unit problem. In N. Wrigley (Ed.), Statistical methods in the spatial sciences. London: Routledge \& Kegan Paul. 127-144.

Openshaw, S., \& Taylor, P. J. (1981). The modifiable areal unit problem. In N. Wrigley, \& R. J. Bennett (Eds.), Quantitative geography: a British view. London: Routledge \& Kegan Paul. 60-69.

Rajabifard, A., Escobar, F., \& Williamson, I. P. (2000). Hierarchical spatial reasoning applied to spatial data infrastructures. Cartography Journal, 29(2), 41-50 (Australia).

Timpf, S., \& Frank, A. U. (1997). Using hierarchical spatial data structures for hierarchical spatial reasoning. In S. Hirtle, \& A. U. Frank (Eds.), Lecture notes in computer science (Vol. 1329) (pp. 69-83). Berlin-Heidelberg: Springer-Verlag.

Tomlin, C. (1992). Geographic information systems and cartographic modeling. Englewood Cliffs: Prentice Hall. 